\title{
Simultaneous primary carcinosarcoma of the uterus and the bilateral fallopian tubes: an extremely rare case and review of the literature
}

\author{
Ali Baloğlu • Cenk Mustafa Güven • Ali Yavuzcan • \\ Burcu Çetinkaya $\cdot$ Seyran Yiğit
}

Received: 16 March 2008/Accepted: 25 July 2008 /Published online: 3 September 2008

(C) Springer-Verlag 2008

\begin{abstract}
Malignant mixed mullerian tumors (MMMTs) are very rare neoplasms of the fallopian tube, consisting of $<1 \%$ of all genital sarcomas. The prognosis of fallopian tube MMMTs is poor. In most cases, the patient is lost in the first 2 years. A case of simultaneous primary carcinosarcoma of the uterus and the bilateral fallopian tubes is reported. Six regimens of carboplatin/paclitaxel combination therapy were administered to the patient after surgery. During the 32-month follow-up of the patient, recurrence and metastasis were not determined. The speculations about the pathological origins and the treatment of MMMTs still continue. Although it is an uncommon occurrence, it has been shown that these tumors may exist simultaneously in different genital organs too.
\end{abstract}

A. Baloğlu • C. M. Güven · A. Yavuzcan • B. Çetinkaya

Gynecology and Obstetric Clinic,

Ataturk Training and Research Hospital 1,

Izmir, Turkey

A. Baloğlu

e-mail: abaloglu@yahoo.com

C. M. Güven

e-mail: drcenkmustafa@yahoo.com

B. Çetinkaya

e-mail: burcumw@yahoo.com

\section{S. Yiğit}

Pathology Department, Ataturk Training and Research Hospital, Izmir, Turkey

e-mail: seyranyigit@hotmail.com

\section{A. Yavuzcan $(\bowtie)$}

Ataturk Egitim ve Arastirma Hastanesi 1,

Kadın Hastalıkları ve Doğum Klinigi,

35820 Izmır, Turkey

e-mail: draliyavuzcan@yahoo.com
Keywords Fallopian tube $\cdot$ Malignant mixed mullarian tumors $\cdot$ Uterus

\section{Introduction}

Malignant mixed mullerian tumors (MMMTs) are metaplastic carcinomas including both sarcomatous and carcinomatous elements [1]. Among the genital organs, uterus and ovary are the organs in which MMMTs are most commonly observed. MMMTs constitute about $2-3 \%$ of the uterus malignancies [2].Sarcomas of the fallopian tube are rarities in gynecologic neoplasms, consisting of $<1 \%$ of all genital sarcomas [3]. The prognosis of fallopian tube MMMTs is poor. In most cases, the patient is lost in the first 2 years.

In this study, we aimed to present a 75 -year-old patient, in whom simultaneous uterus and bilateral fallopian tube MMMTs were determined.

\section{Case report}

A 75-year-old multipara, in menopause since 25 years, applied to our clinic with the complaints of pelvic pain and vaginal bleeding.

The gynecological examination showed a normal vulva, perineum, vagina, and cervix. But a right adnexial mass about $5 \times 5 \mathrm{~cm}$ in diameter was palpated. Routine laboratory tests were normal. Ca-125 value was $64 \mathrm{IU} / \mathrm{ml}$.

Transvaginal ultrasonography (USG) revealed a simple cystic right ovarian tumor, $4 \times 4 \mathrm{~cm}$ in diameter, and a heterogeneous formation in the uterine cavity. The lesions detected by USG were confirmed by abdomino-pelvic computerized tomography (CT). 
Endometrial biopsy was performed, and MMMT was reported after histopathological investigation. Laparatomy revealed normal uterus, bilateral tubes, and left ovary. However, a homogenous cystic mass, $4 \times 4 \mathrm{~cm}$ in diameter, was present in the right adnexial region. No pathologies were detected in the remaining abdominopelvic organs. Ascites was absent. Initially, right oophorectomy was performed, and the sample was sent for frozen section analysis. The result received was benign serous cyst. Hysterectomy, bilateral salphingo-oophorectomy, appendectomy, total omentectomy, and pelvic-paraaortic lymph node dissection were performed. Washings from the pelvis, abdominal gutters, and diaphragmatic recesses were obtained, and multiple biopsies of the peritoneum in the central and lateral pelvis and in the abdomen were done.

Samples were collected from the peritonea and intraperitoneal fluid for the histopathological examination. Tumor metastasis to the intraabdominal organs and tissues was not determined. First, intraperitoneal washing sample was taken for histopathological examination, and mass was excised by total abdominal hysterectomy and bilateral salphingoooferectomy.

The uterus measured $8 \times 5 \times 4 \mathrm{~cm}$. Papillary, fragile tumor tissue was observed in the uterine cavity that was opened lengthwise through the cervical channel. Pathological investigation showed that the tumor contained epithelial (carcinomatous) and mesenchymal (sarcomatous) components (Fig. 1). The sarcomatous component in the uterus tumor consisted of spindle cells with high mitotic activity (Fig. 2). The epithelial component of the tumor in the same location had a papillary growth pattern. Carcinomatous cells had huge, vesicular nuclei and distinct nucleoli. The tumor in the endometrium infiltrated into less than half of the myometrium, and lymphovascular invasion was not present.

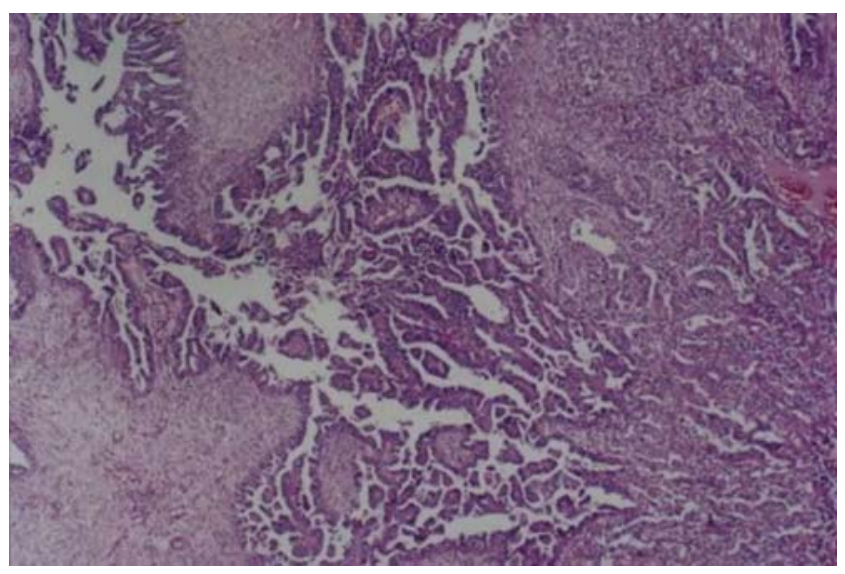

Fig. 1 Both of the carcinomatous and sarcomatous areas of the tumor that have papillary growing pattern in uterus (hematoxylin-eosin $\times 440$ )
The right tube measured $8 \times 1 \mathrm{~cm}$ and the left one measured $6 \times 1 \mathrm{~cm}$. The cut surfaces of both tubes showed a tumoral appearance. Carcinomatous areas demonstrating a papillary growth pattern and sarcomatous areas formed by pleomorphic spindle cells were determined in the samples collected from the tubes. Chondromatous areas were present in the sarcomatous components of the left tube (Fig. 2). Tumor was not present in the serosas of both tubes. Lymphovascular involvement was not observed in the myosalpinx and mesosalpinx. The transition areas between tubal epithelium and neoplastic epithelium were clearly observed (Fig. 3).

HE, PAS, DPAS, and reticulin histochemical stains were applied to the samples collected from the uterus and the tubes. Additionally, vimentin and pancytokeratin (MNF116) staining was performed using strep-avidin-biotin complex method. Cytokeratin gave a positive reaction in the tumor epithelial areas, while vimentin gave a positive reaction in the mesenchymal areas.

The patient was diagnosed with MMMTs, which simultaneously developed in the uterus and bilateral tubes. The tumor in the left tube was MMMT with heterologous component. Metastasis was not present in both ovaries, omentum, and pelvis paraaortic lymph nodes. Malignancy was not determined in the intraperitoneal washing samples or peritoneal biopsies. Both tumors were considered as stage IB according to the FIGO staging system.

Six regimens of carboplatin/paclitaxel combination therapy were administered to the patient in whom postoperative complication did not develop. During the 32-month follow-up of the patient, recurrence and metastasis were not detected, and also, CA125 levels did not increase.

\section{Discussion}

The fallopian tube cancers constitute $0.3 \%$ of the female genital cancers. Mainly, tube cancers are seen in women between the ages of 55 and 60 . Nearly all cancers are of epithelial origin and generally of serous histological type [2]. Fallopian tube MMMTs constitute less than $1 \%$ of genital sarcomas. They may include homologous or heterologous components as sarcomatous elements. Condromatose areas, as in our study, are generally determined as heterologous structures in these tumors [1]. The tumor spread at the time of diagnosis and the residual tumor volume after the surgery have been shown as the most important prognostic factors [4].

The typical symptom triad of fallopian tube cancer is made of watery vaginal discharge, pelvic pain, and pelvic mass. However, this triad is determined only in $15 \%$ of the patients [2]. Accordingly, our patient was complaining from the pain in the right lower abdominal region and had been experiencing vaginal bleeding since 3 months. 
Fig. 2 a The high mitotic activity in the sarcomatous area of uterus and $\mathbf{b}$ the chondromatous area in the sarcomatous places in the tube (hematoxylin-eosin $\times 440$ )
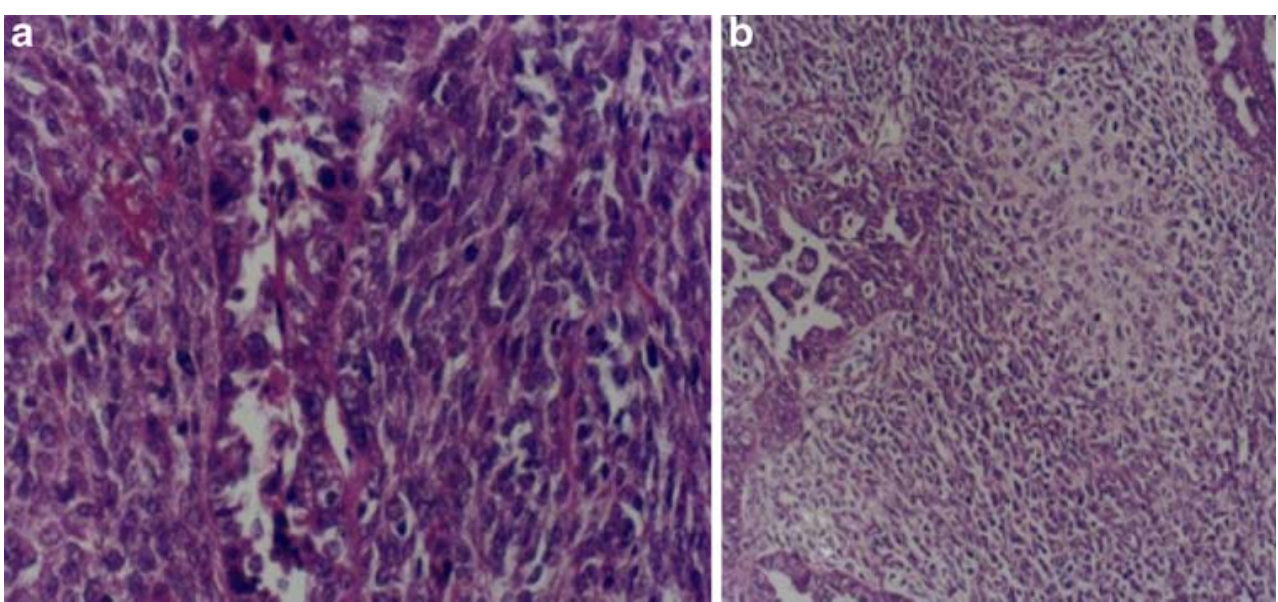

Since they usually are not suspected preoperatively, the diagnosis and treatment are delayed in primary tube cancers. Horn et al. reported that they could diagnose only $25 \%$ of the patients preoperatively [4]. Ayhan et al. could not make a diagnosis in eight patients preoperatively [5]. The presence of a pelvic mass and increased CA-125 levels commonly suggest ovary cancer in a patient. Unfortunately, the diagnosis of primary fallopian tube cancer remains controversial.

The tubal malignancies are mostly thought to be metastatic tumors originating from other organs. Certain criteria have been introduced for primary tubal carcinoma: (1) The main tumor is in the tube and arises from the endosalpinx. (2) Histologically, the pattern reproduces the epithelium of the mucosa and often shows a papillary pattern. (3) If the wall is involved, the transition between benign and malignant epithelium should be demonstrable. (4) The ovaries and endometrium are either normal or contain less tumor than the tube [6]. In our case, in accordance with these criteria, tumor was present macro-

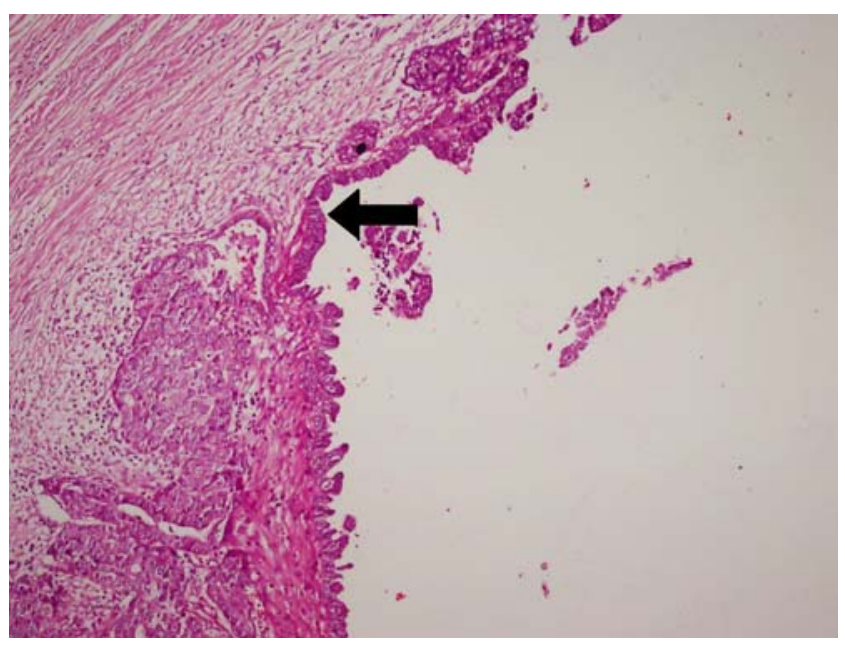

Fig. 3 The transition area between the tubal epithelium and the neoplastic epithelium (hematoxylin-eosin $\times 440$ ) scopically and microscopically in the tube, the tumor showed a papillary pattern, the transition between benign and malignant epithelium was distinctly demonstrated, and malignancy was not present in the ovaries. Additionally, the tumor in the endometrium infiltrated into less than half of the myometrium. There are also some criteria for secondary tubal malignancies introduced by Finn and Javert [7]. According to their criteria, the pervasive and late-stage cancer should primarily be present in the ovary or the endometrium. Additionally, the tube's serousal surface and lymphatics in the myosalpinx and mesosalpinx should be involved [7]. In our case, the stage of the tumor in the endometrium was $1 \mathrm{~B}$ according to the FIGO staging system, and tumor was not determined in the lymphovascular areas of both the uterus and the tube. Due to these criteria, the tumor in the tube was considered as FIGO stage 1B primary tubal carcinosarcoma.

In the recent years, the debates on the preoperative diagnosis of tubal carcinoma have intensified. Kurjak used a 3D, static, power Doppler ultrasonography device for a preoperative diagnosis and reported that findings like papillary protrusion/pseudosepta could be detected by 3D USG. Moreover, Kurjak has also shown that 2D ultrasonography devices are unable to detect bilateral lesions [8]. Transvaginal color Doppler USG shows low vascular impedance [the resistance index (RI) range is between 0.29 and 0.40 ] in fallopian cancers [9]. Using transvaginal color Doppler ultrasonography for the diagnosis of fallopian tube malignancies is more advantageous compared to the other costly procedures [9]. In our case, 2D USG and CT could not detect intratubal lesions; however, it provided correct information about the ovary cyst and the intrauterine lesion.

MMMTs are mostly located in the uterus. Nearly all tumors appear during menopause, at about the age of 62 . Their most common symptoms are postmenopausal bleedings and the enlargement of uterus. These tumors are associated with medical conditions such as obesity, diabe- 
tes, and hypertension. Seven to $37 \%$ of the patients have a history of pelvic radiation exposure [10]. Since the tumors may cause a polypoid protrusion into the uterine cavity, endometrial biopsy turns out to be a method that facilitates the diagnosis in some cases. However, until the diagnosis is made, in $40-60 \%$ of the patients, the disease already is spread out of the uterus [10].

As for the primary synchronous genital invasive neoplasms, they are extremely rare. Thus, established literature information about conduct in these tumors is not present. According to us, the treatment and conduct of each tumor should be handled individually after the diagnosis. Eisner has shown that synchronized endometrial and ovarian cancers are the most common simultaneous genital cancers [11]. The prognosis is better in synchronous uterine and ovarian cancers because the symptoms of the uterine cancer warn the patients and the doctors earlier than the symptoms of the ovarian cancer alone [11]. As a result, the tumors are detected in the early stages. Similarly, the main complaint that led our patient to apply to a doctor was vaginal bleeding due to endometrial cancer.

The treatment of both the fallopian tubes and uterine MMMTs is a combination of surgery, chemotherapy, and radiotherapy. Carlson has shown that the combined use of adjuvant chemotherapy and radiotherapy yields a successful result in tubal sarcomas [12]. The surgical procedure is optimal cytoreductive surgery, including total abdominal hysterectomy and bilateral salpingo-oophorectomy. Gagner has reported that adjuvant radiotherapy performed after the surgery prolongs the mean lifespan [13].Combinations including doxorubicin are more effective in the treatment of MMMTs [10]. Local recurrence may be decreased by radiotherapy in the early stages of uterine MMMTs, but Manes et al. reported that these neoplasms are relatively radioresistant [14]. Radiotherapy is not valid for distant metastases.

Although the preoperative diagnosis of tubal carcinoma is difficult, clinicians should consider primary fallopian tube cancer even if the symptoms are typical. As the appropriate diagnosis is made, the treatment to be performed is adjuvant platinum-based combination chemotherapy after an aggressive cytoreductive surgery [4]. The effectiveness rate of the use of platinium- and paclitaxelbased chemotherapy is $59 \%$ for stages I and II, and $19 \%$ for stages III and IV, according to the FIGO staging system [15].

There are case series and case reports about genital and extragenital MMMTs in the literature $[1,3,16]$. MMMTs of the fallopian tube are also very rare neoplasms. MMMT of the fallopian tube containing immature smooth muscle cells or cells with the myofibroblast-like immunohistochemical phenotype in the undifferentiated component was reported before [17]. Also, a MMMT of the fallopian tube, clinically FIGO stage III, was treated by Das et al. [18]. Table 1 is depicting the presentation and the pathological properties of all the previous reported cases of MMMT of the fallopian tube $[1,3-4,12-14,17-26]$.On the other hand, the synchronous presentation of endometrial and ovarian carcinomas of the endometrioid type is well described; little is known about a similar phenomenon involving the endometrium and fallopian tube. A poorly differentiated synchronous adenocarcinoma of the fallopian tube and the uterus with bilateral ovarian masses was reported [27]. Also, Culton et al. studied 13 patients with synchronous carcinomas of the uterus and fallopian tube, but all of them were endometrioid carcinoma except one. They could not find any synchronous carcinosarcoma of the uterus and fallopian tube like our case [28].

Table 1 Table depicting the presentation and the pathological properties of all the previous reported cases of MMMT of the fallopian tube

\begin{tabular}{lll}
\hline Author/year & Number of cases & Presentation/pathological property \\
\hline Manes and Taylor (1976) [14] & 4 & Carcinosarcomas with hydrosalpinx \\
Buchino and Buchino (1987) [19] & 1 & Homologous-type MMMT \\
Muntz et al. (1989) [20] & 4 & One homologous-type/three heterologous-type MMMT \\
Chang et al. (1991) [21] & 1 & With positive vaginal cytology \\
van Dijk et al. (1990) [22] & 2 & Bilateral/heterologous-type MMMT \\
Imachi et al (1992) [1] & 2 & Heterologous-type MMMT \\
Carlson et al. (1993) [12] & 5 & Heterologous-type MMMT \\
Horn LC et al.(1996) [4] & 4 & Homologous-type MMMT \\
Ebert et al. (1998) [3] & 1 & Five-year survival after minimal surgery without adjuvant treatment \\
Lim et al. (2004) [23] & 1 & With multiple distinct heterologous components \\
Maitra et al. (2004) [24] & 1 & MMMT with Peutz-Jegher's syndrome \\
Gagner and Mittal (2005) [13] & 1 & MMMT as an intraepithelial carcinoma \\
Das et al. (2005) [18] & 1 & Heterologous-type MMMT \\
Hudelist et al. (2006) [25] & 1 & Heterologous-type MMMT \\
Kuroda et al. (2007) [17] & 1 & Coincident with a primary serous carcinoma of the ovary \\
Nappi et al. (2007) [26] & 1 &
\end{tabular}


However, the speculations about the pathological origins and the treatment of MMMTs still continue. Although it is an uncommon occurrence, it has been shown that these tumors may exist simultaneously in different genital organs too.

\section{References}

1. Imachi M, Tsukamoto N, Shigematsu T, Watanabe T, Uehira K, Amada $\mathrm{S}$ et al (1992) Malignant mixed mullerian tumor of the fallopian tube. Report of two cases and review of literature. Gynecol Oncol 47:114-124

2. Berek JS, Hacker NF (2000) Practical gynecologic oncology, 3rd edn. Lippincott Williams \& Wilkins, Baltimore, MD, p 208

3. Ebert AD, Perez-Canto A, Schaller G, Entezami M, Hopp HS, Weitzel HK (1998) Stage I primary malignant mixed müllerian tumor of the fallopian tube. Report of a case with five-year survival after minimal surgery without adjuvant treatment. J Reprod Med 43:598-600

4. Horn LC, Werschnik C, Bilek K, Emmert C (1996) Diagnosis and clinical management in malignant mullerian tumors of the fallopian tube. Arch Gynecol Obstet 258:47-53

5. Ayhan A, Deren O, Yuce K, Tuncer Z, Mocan G (1994) Primary carcinoma of the fallopian tube: a study of 8 cases. Eur $\mathrm{J}$ Gynaecol Oncol 15:147-151

6. Fox H, Wells M (2003) Haines and Taylor obstetrical and gynaecological pathology, 5th edn. Elsevier Science Limited, Churcill Livingstone, p 605

7. Fox H, Wells M (2003) Haines and Taylor obstetrical and gynaecological pathology, 5th edn. Elsevier Science Limited, Churcill Livingstone, p 615

8. Kurjak A, Kupesic S, Jacobs I (2000) Preoperative diagnosis of the primary fallopian tube carcinoma by three-dimensional static and power Doppler sonography. Ultrasound Obstet Gynecol 15:246-251

9. Kurjak A, Kupesic S, Ilijas M, Sparac V, Kosuta D (1998) Preoperative diagnosis of primary fallopian tube carcinoma. Gynecol Oncol 68:29-34

10. Berek JS (1996) Novak's gynecology, 12th edn. Lippincott Williams \& Wilkins, Baltimore, MD, p 1096

11. Eisner RF, Nieber RK, Berek JS (1989) Synchrous primary neoplasms of the female reproductive tract. Gynecol Oncol 33:335-339

12. Carlson JA Jr, Ackerman BL, Wheeler JE (1993) Malignant mixed mullerian tumor of the fallopian tube. Cancer 71:187-192

13. Gagner JP, Mittal K (2005) Malignant mixed mullerian tumor of the fimbriated end of the fallopian tube: origin as an intraepithelial carcinoma. Gynecol Oncol 97:219-222
14. Manes JL, Taylor HB (1976) Carcinosarcoma and mixed müllerian tumors of the fallopian tube: report of four cases. Cancer 38:1687-1693

15. Barakat RR, Rubin SC, Saigo PE, Lewis JL Jr, Jones WB, Curtin JP (1992) Second-look laparatomy in carcinoma of the fallopian tube. Obstet Gynecol 82:748-751

16. Mikami M, Kuwabara Y, Tanaka K, Komiyama S, Ishikawa M, Hirose T (2005) Malignant mixed müllerian tumor of primary mesenteric origin. Int J Gynecol Cancer 15:1249-1253

17. Kuroda N, Inui Y, Ohara M, Hirouchi T, Mizuno K, Kubo A et al (2007) Hyaline globule-like structures in undifferentiated sarcoma cells of malignant müllerian mixed tumor of the fallopian tube. Med Mol Morphol 40:46-49

18. Das TK, Raha K, Bandyopadhyay A, Dasgupta A, Ghosh D, Mondal AK (2005) Malignant mixed mullerian tumour of the fallopian tube of heterologous variety - a case report. Indian J Pathol Microbiol 48:354-356

19. Buchino JJ, Buchino JJ (1987) Malignant mixed müllerian tumor of the fallopian tube. Arch Pathol Lab Med 111:386-387

20. Muntz HG, Rutgers JL, Tarraza HM, Fuller AF Jr (1989) Carcinosarcomas and mixed müllerian tumors of the fallopian tube. Gynecol Oncol 34:109-115

21. Chang HC, Hsueh S, Soong YK (1991) Malignant mixed müllerian tumor of the fallopian tube. Case report and review of the literature. Changgeng Yi Xue Za Zhi 14:259-263

22. van Dijk CM, Kooijman CD, van Lindert AC (1990) Malignant mixed müllerian tumour of the fallopian tube. Histopathology $16: 300-302$

23. Lim BJ, Kim JW, Yang WI, Cho NH (2004) Malignant mixed müllerian tumor of fallopian tube with multiple distinct heterologous components. Int J Gynecol Cancer 14:690-693

24. Maitra RN, Lee J, McConnell DT, Kenwright DN, Dady P (2004) Malignant mixed mullerian tumour of the fallopian tube occurring in a patient with Peutz-Jegher's syndrome. Aust N Z J Obstet Gynaecol 44:77-79

25. Hudelist G, Unterrieder K, Kandolf O, Alpi G, Pucher S, Pollak G, Czerwenka K, Keckstein J (2006) Malignant mixed Müllerian tumor with heterologous component arising in the fallopian tube - a case report. Eur J Gynaecol Oncol 27:509-512

26. Nappi L, Indraccolo U, Matteo M, Rosenberg P, Greco P (2007) Malignant mixed müllerian tumor of the fallopian tube coincident with a primary serous carcinoma of the ovary. Case report. Eur J Gynaecol Oncol 28:511-512

27. Junaid I, Paz R, Salihu HM, Sharma PP, Aliyu ZY (2006) PseudoMeig's syndrome with multiple synchronous benign and malignant pelvic tumors. Arch Gynecol Obstet 273:315-318

28. Culton LK, Deavers MT, Silva EG, Liu J, Malpica A (2006) Endometrioid carcinoma simultaneously involving the uterus and the fallopian tube: a clinicopathologic study of 13 cases. Am J Surg Pathol 30:844-849 